\title{
Association between Coagulation Function and Cerebral Microbleeds in Ischemic Stroke Patients with Atrial Fibrillation and/or Rheumatic Heart Disease
}

\author{
Junfeng Liu, Deren Wang, Yao Xiong, Bian Liu, Jing Lin, Shihong Zhang, Bo Wu, Chenchen \\ Wei, Ming Liu*
}

Stroke Clinical Research Unit, Department of Neurology, West China Hospital, Sichuan University, Chengdu, 610041 China

[Received May 23, 2016; Revised July 1, 2016; Accepted July 15, 2016]

\begin{abstract}
Cerebral microbleeds (CMBs), which indicate hemorrhage-prone disease, may associate with hemostatic abnormalities, but the association between CMBs and coagulation function is uncertain. We aimed to examine this possible association. The following coagulation function indicators were evaluated in 85 consecutive ischemic stroke patients diagnosed with atrial fibrillation and/or rheumatic heart disease: prothrombintime (PT), activated partial thromboplastin time (APTT), and levels of D-dimer and fibrinogen. Indicators were assessed within $24 \mathrm{~h}$ after admission. CMBs were identified based on published criteria by two experienced stroke neurologists working independently. PT, APPT, and levels of D-dimer and fibrinogen were compared between patients with and without CMBs using univariate and multivariate analysis. CMBs were detected in 48 patients $(56.5 \%)$, and fibrinogen levels in these patients were independently and significantly higher than in patients without CMBs after adjustment (OR 2.16, 95\% CI 1.20-3.90, $P=0.01$ ), whereas the two types of patients did not differ significantly in PT, APPT, or D-dimer levels. The presence of CMBs in ischemic stroke patients with atrial fibrillation and/or rheumatic heart disease is associated with elevated levels of fibrinogen. Larger prospective studies are needed to verify this association and explore the mechanisms involved.
\end{abstract}

Key words: Coagulation function, Fibrinogen, Cerebral microbleeds, Ischemic stroke

Cerebral microbleeds (CMBs) are one kind of cerebral small vessel disease [1]. They manifest as small, round lesions showing signal loss on $\mathrm{T} 2 *$-weighted gradient echo (GRE) or susceptibility-weighted magnetic resonance imaging, which is due to perivascular hemosiderin [2]. Strictly lobar CMBs are associated with cerebral amyloid angiopathy, and deep CMBs are associated with hypertensive arteriopathy [3-5].

CMBs occur in many patients with ischemic stroke, and primary intracerebral hemorrhage (ICH); $\mathrm{CMBs}$ are also associated with elevated risk of spontaneous $\mathrm{ICH}$, and symptomatic ICH after intravenous thrombolysis [68]. CMBs are thought to indicate hemorrhage-prone disease in the brain [9], but whether such bleeding is associated with hemostatic abnormalities or vascular lesions needs to be clarified.

Several studies have suggested a link between CMBs and coagulation dysfunction. Prodan et al [10] found that the presence of CMBs in patients with non-lacunar ischemic stroke is associated with lower levels of coatedplatelets, which are involved in thrombin generation and thereby help drive coagulation [11]. CMBs were

*Correspondence should be addressed to: Dr. Ming Liu, Stroke Clinical Research Unit, Department of Neurology, West China Hospital, Sichuan University, Chengdu, 610041, Sichuan Province, China. E-mail: wyplmh@hotmail.com

Copyright: () 2016. This is an open-access article distributed under the terms of the Creative Commons Attribution License (CC BY 4.0), which permits unrestricted use, distribution, and reproduction in any medium, provided the original author and source are credited. 
identified in a 62-year-old Spanish male with disseminated intravascular coagulation, a serious disease related to coagulation dysfunction [12]. These sporadic reports suggest an association between CMBs and compromised coagulation function, but more systematic studies are needed.

Therefore, the present research was undertaken to compare ischemic stroke patients with and without CMBs in terms of several indicators of coagulation function, including prothrombin time, activated partial thromboplastin time, and levels of D-dimer and fibrinogen.

\section{MATERIAS AND METHODS}

This research was conducted using data from the National Natural Science Foundation of China project "Study on small vessel pathological mechanism of cerebral hemorrhage after cardioembolic stroke using susceptibility-weighted MR imaging markers', The research was approved by the Medical Ethics Committee of West China Hospital, Sichuan University. Written informed consent was obtained from participants or their next of kin.

The present study prospectively enrolled consecutive ischemic stroke patients with atrial fibrillation and/or rheumatic heart disease who were admitted to West China Hospital of Sichuan University (Chengdu, China) between January 2014 and March 2016, within one month after stroke onset. To participate in the study, patients had to undergo complete susceptibility-weighted magnetic resonance imaging within 7 days after admission. The diagnosis of ischemic stroke, made based on World Health Organization criteria [13], had to be confirmed based on computed tomography scanning or magnetic resonance imaging [14]. Atrial fibrillation was defined as a history of persistent or paroxysmal atrial fibrillation, documented by electrocardiography during admission (24-hour or not) [15]. Rheumatic heart disease was diagnosed according to criteria in the International Classification of Diseases (10th edition) and confirmed by echocardiography [16]. Patients were excluded from the study if their coagulation function was not analyzed within $24 \mathrm{~h}$ after admission by measuring prothrombintime (PT), activated partial thromboplastin time (APTT), and levels of D-dimer and fibrinogen.

A standardized form was used to collect the following patient information: demographic characteristics (age and gender); risk factors (hypertension, diabetes mellitus, hyperlipidemia, history of transient ischemic attacks and stroke, current smoking and alcohol consumption); stroke severity on admission (score on the National Institutes of Health Stroke Scale [NIHSS]) [17]; and renal impairment, which was defined as an estimated glomerular filtration rate $<60 \mathrm{ml} / \mathrm{min} / 1.73 \mathrm{~m}^{2}$ [18-19], based on medical history or prospective measurement.

Coagulation function was assessed using routine laboratory analysis of blood samples taken within $24 \mathrm{~h}$ after admission. Levels of D-dimer and fibrinogen were measured using, respectively, acalibrated SYSMEX7000 analyzer (Sysmex Corporation, Hyogo, Japan) and STA$\mathrm{R}$ analyzer (Evolution, Stago, France). The reference range for D-dimer was $<0.55 \mathrm{mg} / \mathrm{L}$ fibrinogen equivalent units (FEU), and the reference range for fibrinogen was 2$4 \mathrm{~g} / \mathrm{L}$. PT, APTT, and levels of serum cholesterol, triglycerides (TG), high-density lipoprotein (HDL), and low-density lipoprotein (LDL) were also measured. All these analyses were performed in the Department of Laboratory Medicine of West China Hospital, Sichuan University, which has been fully accredited by the Chinese Ministry of Health and the College of American Pathologists' Laboratory Accreditation Program [20].

All participants were imaged by fluid-attenuated inversion recovery and susceptibility-weighted magnetic resonance imaging at the Huaxi MR Research Center within 7 days after admission using a dedicated 3-T imaging system (Siemens Trio). The following operating parameters were used to perform susceptibility-weighted magnetic resonance imaging, and they remained unchanged throughout the study: type of echo sequence, multislice gradient; repetition time, $207 \mathrm{~ms}$; echo time, 20 $\mathrm{ms}$; flip angle, $15^{\circ}$; slice thickness, $2 \mathrm{~mm}$; number of slices, 60; interslice gap, $0 \mathrm{~mm}$; coverage, entire brain; field of view, $220 \times 173 \mathrm{~mm}$; matrix, $256 \times 256$. The following operating parameters were used to perform fluid-attenuated inversion recovery magnetic resonance imaging, and they remained unchanged throughout the study: repetition time, $6000 \mathrm{~ms}$; echo time, $100 \mathrm{~ms}$; flip angle, $90^{\circ}$; slice thickness, $5 \mathrm{~mm}$; number of slices, 21 ; interslice gap, $1.5 \mathrm{~mm}$; field of view, $220 \times 200 \mathrm{~mm}$; matrix, $256 \times 256$.

CMBs were defined as small, circular, homogeneous lesions appearing throughout the brain with very low signal intensity and diameter $<10 \mathrm{~mm}$ on susceptibilityweighted magnetic resonance images [5,21]. CMBs were classified based on location as (a) strictly lobar CMBs (b) deep or infratentorial CMBs with or without concomitant lobar CMBs [2,3]. Two neurologists blinded to clinical data independently assessed each patient for the presence of CMBs. A third neurologist arbitrated in case of disagreement, and a consensus decision was reached. Inter-rater reliability for determining the presence and location of CMBs was 0.73 .

Data for continuous variables are reported as mean \pm standard deviation or as median (interquartile range [IQR]), while data for categorical variables are reported as frequencies and percentages. Statistical significance of inter-group differences was assessed using the $\chi^{2}$ test, 
Fisher's exact test (categorical data), or the $t$ test or MannWhitney U test (continuous data), as appropriate. Possible correlations of $\mathrm{CMB}$ occurrence with patient variables and/or coagulation function were explored using multivariate logistic regression. When appropriate, results were reported using odds ratios (ORs) and 95\% confidence intervals (95\%CIs). All data were analyzed using The Statistical Package for the Social Sciences 20.0 (SPSS; IBM, Chicago, IL, USA). The threshold of significance was defined as $p<0.05$.

\section{RESULTS}

The study enrolled a consecutive sample of 109 ischemic stroke patients, along with atrial fibrillation and/or rheumatic heart disease, who were admitted to our hospital within one month of stroke onset and underwent susceptibility-weighted magnetic resonance imaging within 7 days after admission. Of these enrolled patients, 24 were excluded because their coagulation function was not analyzed within $24 \mathrm{~h}$ after admission. The remaining 85 patients $(35.3 \%$ male) were included in the final analysis. Their mean age was $68.96 \pm 11.90 \mathrm{yr}$ (ranges from 42 to 92). Most patients (67) had only atrial fibrillation, 4 of them had only rheumatic heart disease, and 14 of them had both conditions. Just over half (48, $56.5 \%$ ) showed evidence of at least one CMB (ranges from 1-10): of these patients, 26 (54.2\%) had strictly lobar CMBs, while the remaining $22(45.8 \%)$ had deep or infratentorial $\mathrm{CMBs}$ with or without concomitant lobar CMBs.

Table1. Baseline characteristics of ischemic stroke patients with atrial fibrillation and/or rheumatic heart disease, stratified by presence or absence of cerebral microbleeds

\begin{tabular}{|c|c|c|c|c|}
\hline Characteristic & $\begin{array}{l}\text { Total } \\
\mathbf{N}=\mathbf{8 5}\end{array}$ & $\begin{array}{l}\text { CMBs } \\
\mathrm{N}=48\end{array}$ & $\begin{array}{l}\text { No CMBs } \\
\mathbf{N}=37\end{array}$ & $p$ \\
\hline Male & $30(35.3)$ & $19(39.6)$ & $11(29.7)$ & 0.35 \\
\hline Age, yr & $68.94 \pm 11.90$ & $70.98 \pm 10.50$ & $66.30 \pm 13.18$ & 0.07 \\
\hline Current smoking & $69(81.2)$ & $39(81.3)$ & $30(81.1)$ & 0.99 \\
\hline $\begin{array}{l}\text { Current alcohol consumption } \\
\text { Hypertension }\end{array}$ & $\begin{array}{l}73(85.9) \\
38(44.7)\end{array}$ & $\begin{array}{l}42(87.5) \\
25(52.1)\end{array}$ & $\begin{array}{l}31(83.8) \\
13(35.1)\end{array}$ & $\begin{array}{l}0.83 \\
0.12\end{array}$ \\
\hline Diabetesmellitus & $25(29.4)$ & $12(25.0)$ & $13(35.1)$ & 0.31 \\
\hline Renal impairment & $15(17.6)$ & $9(18.8)$ & $6(16.2)$ & 0.76 \\
\hline Previous TIA/stroke & $17(20.0)$ & $9(18.8)$ & $8(21.6)$ & 0.74 \\
\hline NIHSS score on admission & $9(4-13)$ & $6.5(3-10)$ & $12(6-16)$ & 0.03 \\
\hline Total cholesterol, mmol/L & $4.03 \pm 0.91$ & $4.12 \pm 0.97$ & $3.91 \pm 0.83$ & 0.29 \\
\hline $\mathrm{TG}, \mathrm{mmol} / \mathrm{L}$ & $1.49 \pm 1.35$ & $1.60 \pm 1.64$ & $1.35 \pm 0.83$ & 0.41 \\
\hline HDL, $\mathrm{mmol} / \mathrm{L}$ & $1.39 \pm 0.39$ & $1.41 \pm 0.40$ & $1.36 \pm 0.38$ & 0.61 \\
\hline LDL, mmol/L & $2.26 \pm 0.80$ & $2.36 \pm 0.87$ & $2.13 \pm 0.69$ & 0.18 \\
\hline PT, s & $12.54 \pm 1.61$ & $12.59 \pm 1.75$ & $12.47 \pm 1.44$ & 0.73 \\
\hline APTT, $\mathbf{s}$ & $28.43 \pm 7.02$ & $29.13 \pm 8.93$ & $27.54 \pm 3.01$ & 0.31 \\
\hline D-dimer, mg/L & $2.51 \pm 5.14$ & $2.73 \pm 5.70$ & $2.22 \pm 4.36$ & 0.65 \\
\hline Fibrinogen, g/L & $3.02 \pm 1.03$ & $3.24 \pm 1.14$ & $2.73 \pm 0.78$ & 0.02 \\
\hline
\end{tabular}

Values are $\mathrm{n}(\%)$, median (IQR) or mean \pm SD.

Abbreviations: APTT, activated partial thromboplastin time; CMBs, cerebral microbleeds; HDL, high-density lipoprotein; hs-cTnT, high-sensitivity cardiac troponin T; LDL, low-density lipoprotein; NIHSS, National Institutes of Health Stroke Scale; PT, prothrombintime; TG,

The characteristics of study participants stratified by presence or absence of CMBs are shown in Table 1, which reports coagulation function indicators as continuous variables. Patients with CMBs were more likely to have a lower NIHSS score on admission than patients without CMBs $(p=0.03)$, as well as a significantly higher level of fibrinogen $(p=0.02)$. Patients with CMBs tended to have longer PT ( $p=0.73)$, longer APTT $(p=0.31)$ and a higher level of D-dimer $(p=0.65)$.

Multivariate analysis was performed using a model that adjusted for age, gender and baseline variables differing significantly between patients with or without CMBs, i.e. NIHSS on admission. The model identified fibrinogen level as an independent risk factor for CMB 
occurrence (OR 2.16, 95\% CI 1.20-3.90, $p=0.01$; Table 2).

Table 2. Multivariate analysis to identify coagulation function indicators independently associated with $\mathrm{CMB}$ occurrence*

\begin{tabular}{llll}
\hline & OR & $\mathbf{9 5 \%}$ CI & $p$ \\
\hline PT, s & 1.06 & $0.79-1.44$ & 0.69 \\
APTT, s & 1.04 & $0.95-1.14$ & 0.37 \\
D-dimer, mg/L & 1.02 & $0.92-1.13$ & 0.74 \\
Fibrinogen, g/L & 2.16 & $1.20-3.90$ & $\mathbf{0 . 0 1}$ \\
\hline
\end{tabular}

*Data wereadjusted for age, gender, and NIHSS score on admission. Abbreviations: APTT, activated partial thromboplastin time; CI, confidence interval; CMB, cerebral microbleeds; NIHSS, National Institutes of Health Stroke Scale; OR, odds ratio; PT, prothrombintime

\section{DISCUSSION}

This prospective study assessed possible relationships between indicators of coagulation function and the presence of CMBs in ischemic stroke patients with atrial fibrillation and/or rheumatic heart disease. Our results suggest that fibrinogen levels are independently associated with risk of CMBs, while PT, APTT and Ddimer levels are not. To our knowledge, this is the first study to examine possible correlations between coagulation function and CMBs.

Why fibrinogen levels may be associated with risk of CMBs is unclear. Fibrinogen, a critical element of platelet cross-linking and clot formation, plays an important role in arterial thrombosis [22]. A study in Japan [23] has linked serum fibrinogen levels with intima-media thickness and atherosclerosis. Furthermore, fibrinogen is generated and released when systemic inflammation occurs [24], and both inflammation and atherosclerosis have been linked to CMB formation [25]. Taken together, these data suggest that elevated fibrinogen levels may increase risk of CMBs through their association with inflammation and atherosclerosis.

The present study involved a relatively small, highly specific patient population, so future prospective studies with larger cohorts are needed to verify and extend our finding of an association between elevated fibrinogen levels and risk of CMB occurrence. Those studies should examine whether and how elevated fibrinogen levels may contribute to $\mathrm{CMB}$ formation as well as to other small cerebral vascular diseases.

\section{Conclusion}

Elevated serum fibrinogen concentration in ischemic stroke patients with atrial fibrillation and/or rheumatic heart disease may be associated with CMB formation. Future studies should verify this association and explore how elevated fibrinogen may contribute to $\mathrm{CMB}$ formation.

\section{Acknowledgments}

This research was supported by the National Natural Science Foundation of China (81371282 and 81400964).

\section{Declaration of interest}

The authors report no conflicts of interest. The authors alone are responsible for writing the paper and the contents therein.

\section{References}

[1] Bath PM, Wardlaw JM (2015). Pharmacological treatment and prevention of cerebral small vessel disease: a review of potential interventions. Int J Stroke,10: 469478.

[2] Fazekas F, Kleinert R, Roob G, Kleinert G, Kapeller P, Schmidt R, et al. (1999). Histopathologic analysis of foci of signal loss on gradient-echo $\mathrm{T} 2 *$-weighted MR images in patients with spontaneous intracerebral hemorrhage: evidence of microangiopathy-related microbleeds. Am J Neuroradiol, 20: 637-642.

[3] Vernooij M, van der Lugt A, Ikram M, Wielopolski PA, Niessen WJ, Hofman A, et al. (2008). Prevalence and risk factors of cerebral microbleeds - the Rotterdam scan study. Neurology, 70:1208-1214.

[4] Benedictus MR, Goos JD, Binnewijzend MA, Muller M, Barkhof F, Scheltens P, et al. (2013). Specific risk factors for microbleeds and white matter hyperintensities in Alzheimer's disease. Neurobiol Aging, 34: 2488-2494.

[5] Poels MM, Vernooij MW, Ikram M, Hofman A, Krestin GP, van der Lugt A, et al. (2010). Prevalence and risk factors of cerebral microbleeds an update of the Rotterdam scan study. Stroke, 41: S103-S106.

[6] Greenberg SM, Eng JA, Ning MM, Smith EE, Rosand J (2004). Hemorrhageburden predicts recurrent intracerebral hemorrhage afterlobar hemorrhage. Stroke, 35: 1415-1420.

[7] Nighoghossian N, Hermier M, Adeleine P, BlancLasserre K, Derex L, Honnorat J, et al. (2002). Old microbleeds are a potential risk factor for cerebral bleeding after ischemic stroke - a gradient-echo T2*weighted brain MRI study. Stroke, 33: 735-742.

[8] Tsivgoulis G, Zand R, Katsanos AH, Turc G, Nolte CH, Jung S, et al. (2016). Risk of Symptomatic Intracerebral Hemorrhage After Intravenous Thrombolysis in Patients with AcuteIschemic Stroke and High Cerebral 
Microbleed Burden: A Meta-analysis. JAMA Neurol, 73: 675-83.

[9] Charidimou A, Kakar P, Fox Z, Werring DJ (2013). Cerebral microbleeds and recurrent stroke risk: systematic review and meta analysis of prospective ischemic stroke and transient ischemic attack cohorts. Stroke, 44: 995-1001.

[10] Prodan CI, Stoner JA, Gordon DL, Dale GL (2014). Cerebral microbleeds in nonlacunarbrain infarction are associated with lower coated-platelet levels. J Stroke Cerebrovasc Dis, 23(5): e325-30.

[11] Dale GL (2005). Coated-platelets: an emerging component of the procoagulant response. J Thromb Haemost, 3:2185-2192.

[12] Trevino-Peinado C, Zubieta JL, Fernández MM (2015). Subcortical Microbleeds in Disseminated Intravascular Coagulation Mimicking Amyloid Angiopathy. J Neuroimaging, 25: 660-1.

[13] Hatano S (1976). Experience from a multicentre stroke register: a preliminary report. Bull World Health Organ, 54: 541-53.

[14] Sacco RL, Kasner SE, Broderick JP, Caplan LR, Connors JJ, Culebras A, et al. (2013). An updated definition of stroke for the 21st century: a statement for healthcare professionals from the American Heart Association/American Stroke Association. Stroke, 44: 2064-89.

[15] Lin S, Wu B, Hao ZL, Kong FY, Tao WD, Wang DR, et al. (2011). Characteristics, treatment and outcome of ischemic stroke with atrial fibrillation in a Chinese hospital-based stroke study. Cerebrovasc Dis, 31: 419426.

[16] International statistical classification of diseases and related health problems 10th revision. World Health Organization. Available at: http://apps. who.int/classifications/icd10/browse/2010/en.

[17] Brott T, Adams HP Jr, Olinger CP, Marler JR, Barsan WG, Biller J, et al. (1989). Measurements of acute cerebral infarction: a clinical examination scale, Stroke, 20: 864-70.

[18] Levey AS, Coresh J, Greene T, Stevens LA, Zhang YL, Hendriksen S, et al. (2006). Using standardized serum creatinine values in the modification of diet in renal disease study equation for estimating glomerular filtration rate. Ann Intern Med, 145: 247-254.

[19] Sarnak MJ, Levey AS, Schoolwerth AC, Coresh J, Culleton B, Hamm LL, et al (2003). Kidney disease as a risk factor for development of cardiovascular disease: a statement from the American Heart Association Councils on Kidney in Cardiovascular Disease, High Blood Pressure Research, Clinical Cardiology, and Epidemiology and Prevention. Circulation, 108: 21542169.

[20] Shen Y, Yang T, Jia L, Wang T, Chen L, Wan C, et al. (2013). A potentialrole for D-dimer in the diagnosis of tuberculous pleural effusion. Eur Rev Med Pharmacol Sci, 17: 201-5.

[21] Greenberg SM, Vernooij MW, Cordonnier C, Viswanathan A, Al-Shahi Salman R, Warach S, et al (2009). Cerebral microbleeds: a guide to detection and interpretation. Lancet Neurol, 8:165-174.

[22] Ang L, Mahmud E (2008). Monitoring oral antiplatelet therapy: is it justified? Ther Adv Cardiovasc Dis, 2: $485 \mathrm{e} 496$.

[23] Kawase Ishihara K, Kokubo Y, Yokota C, Hida E, Miyata T, Toyoda K, et al. (2015). Effect of Plasma Fibrinogen, High-SensitiveC-Reactive Protein, and Cigarette Smoking on Carotid Atherosclerosis: The Suita Study. J Stroke Cerebrovasc Dis, 242385-9.

[24] Smith EB (1995). Fibrinogen, fibrin and the arterial wall, Eur. Heart J, 16 (Suppl. A):11-4.

[25] Shoamanesh A, Preis SR, Beiser AS, Vasan RS, Benjamin EJ, Kase CS, et al. (2015). Inflammatory biomarkers, cerebral microbleeds, and small vessel disease: Framingham Heart Study. Neurology, 84: 82532 . 